\title{
Diffuse radio sources in clusters of galaxies: models and long-wavelength radio observations
}

\author{
Craig L. Sarazin \\ Department of Astronomy, University of Virginia, Charlottesville, VA 22903, USA \\ email: sarazin@virginia.edu
}

\begin{abstract}
Clusters of galaxies contain several types of diffuse radio sources with very steep radio spectra which are associated with the cluster environment, including central radio bubbles, cluster radio relics, and cluster radio halos. Radio halos and relics are found only in merging clusters. Cluster radio relics may be produced by particle acceleration in merger shocks, while radio halos, may result from electron re-acceleration by turbulence produced by mergers. Secondary production of electrons and positrons by hadronic interactions also plays a role. If cluster radio halos and relics are related to mergers, then deep low frequency radio surveys could detect 1000 's of clusters. Long-wavelength radio observations have a great potential to help us understand clusters and large scale structure, and can provide a diagnostic of cluster mergers, which affect the use of clusters in cosmological and dark energy studies.
\end{abstract}

Keywords. shock waves, galaxies: clusters: general, cooling flows, cosmological parameters, intergalactic medium, radio continuum: galaxies, X-rays: galaxies: clusters

\section{Introduction}

Clusters of galaxies contain several types of radio sources which are associated with the cluster environment. First, the $\mathrm{cD}$ galaxies at the centers of cooling core clusters almost always have small, spatially distorted, mainly FR I radio sources; the radio lobes of these sources often appear to be displacing the X-ray emitting cluster gas, producing holes in the X-ray emission surrounded by bright shells (e.g., Blanton et al. 2001). Second, there are cluster radio halo sources, which tend to be relatively symmetric and centrally located, are not strongly polarized, and can be $\sim \mathrm{Mpc}$ in size (e.g., Feretti et al. 2004). Third, there are cluster radio relic sources, which are often elongated, are generally located in the periphery of clusters, are often strongly polarized, and sometimes occur in pairs on opposite sides of the cluster center (e.g., Giovannini \& Feretti 2004). Both cluster radio halos and radio relics are not associated with individual radio galaxies, and only occur in merging clusters. Finally, there are centrally located 'radio relics,' which often are filamentary; recent radio and X-ray observations suggest that they may be radio lobes from the central $\mathrm{cD}$ galaxies, displaced by buoyancy or motions in the cluster (Fujita et al. 2002). The diffuse potions of all of these cluster radio sources tend to have rather steep radio spectra; in part, this is due to the fact that they are confined by the high pressure, X-ray emitting gas in clusters, and thus suffer radiative losses rather than adiabatic expansion.

\section{Mergers and radio halos and relics}

Clusters of galaxies form hierarchically through the gravitational merger of smaller clusters and groups. Major cluster mergers are the most energetic events in the Universe 
since the Big Bang, releasing gravitational binding energies of as much as $\sim 10^{64} \mathrm{erg}$. Mergers drive shocks into the intracluster medium, and generate turbulence. The shocks and turbulence may accelerate or re-accelerate relativistic particles; the relativistic electrons will emit synchrotron radio emission in the intracluster magnetic field. The electrons which produce radio emission at most observable wavelengths have energies of many $\mathrm{GeV}$, and have relatively short lifetimes $\left(\sim 10^{8} \mathrm{yr}\right)$ in clusters due to synchrotron and inverse Compton (IC) emission. Thus, radio-emitting electrons accelerated in a mergers will tend to survive only for a time scale which is comparable to the duration of the merger. Ions have much longer lifetimes in clusters, and the total input of relativistic hadrons in a cluster from mergers and other sources (e.g., AGNs) will tend to accumulate over the lifetime of the cluster. Thus, another source of relativistic electrons in the intracluster medium is secondary production by hadrons. For example, relativistic protons will collide with thermal protons in the intracluster medium and generate pions and other mesons, $p+p \rightarrow p+p+n \pi$, and the charged pions will decay to produce electrons and positrons, $\pi^{ \pm} \rightarrow \mu^{ \pm}+\nu_{\mu}\left(\bar{\nu}_{\mu}\right), \mu^{ \pm} \rightarrow e^{ \pm}+\nu_{e}\left(\bar{\nu}_{e}\right)+\bar{\nu}_{\mu}\left(\nu_{\mu}\right)$. Secondary production will give a steady source of relativistic electrons which should be present in all clusters; the fact that bright radio halos and relics are only found in merging clusters suggests that the bulk of the radio emitting electrons are accelerated during the merging.

Two mechanisms by which cluster mergers can lead to the (re-)acceleration of relativistic particles are shock acceleration and turbulent acceleration. It seems most likely that shock acceleration is the mechanism for producing cluster radio relics. They are elongated, often located at positions which are sensible locations for merger shocks in their host clusters, often have a sharp edge (presumably the side of the relic at the shock), and sometimes have radio spectra which steepen away from the shock edge (as might be expected due to radiative losses by the accelerated electrons) (e.g., Giovannini \& Feretti 2004; Clarke \& Ensslin 2006). An alternative model for radio relics is that they start as 'radio ghosts', or old radio plasma from a radio galaxy in which the high energy electrons have lost most of their energy. A merger shock could adiabatically compress such a radio ghost, and reactivate it as a visible radio source (Ensslin \& Gopal-Krishna 2001).

On the other hand, cluster radio halos are unlikely to be produced directly by merger shock acceleration of electrons. The halos are large and fairly symmetrical, and they extend too far from merger shocks for the radio emitting electrons to have moved without losses. Thus, halos appear to require a distributed source of relativistic electrons. This might be secondary production, or turbulent acceleration following the passage of merger shocks (e.g., Fujita et al. 2003; Cassano \& Brunetti 2005). For turbulent re-acceleration, one needs a reservoir of low energy, relativistic electrons; these have long lifetimes in clusters, so they may be due to previous mergers and/or AGN activity. One also requires that post-merger regions of merging clusters have turbulence with an energy density of $\sim 20 \%$ of the thermal energy density of the intracluster gas; this agrees reasonably with the results from numerical simulations (Ricker \& Sarazin 2001).

While all the clusters which have so far been observed to contain cluster radio relics or halos are merging clusters, it certainly is not the case that all merging clusters are observed to have diffuse radio emission. However, it is clear that all of the existing surveys for diffuse radio sources in clusters are strongly limited by their surface brightness sensitivity. Because of their steep radio spectra, we need very deep, low frequency radio surveys to detect the bulk of merging clusters (probably 1000's). 


\section{Clusters and cosmology}

Clusters of galaxies are very important cosmological probes, and X-ray or SunyaevZeldovich (SZ) surveys of clusters are likely to be used in the next few years to study cosmology and dark energy (Mohr 2005). All of these surveys require estimates of the masses of the clusters, which are usually based on their X-ray or SZ properties assuming equilibrium. Cluster mergers pose a problem for these surveys, since during a merger the X-ray luminosity and temperature and SZ effect are considerably boosted, and the clusters are not in hydrostatic equilibrium (Randall et al. 2002; Wik et al. 2006).

In principle, self-calibration (Mohr 2005) may deal with these problems, but it might be better if a simple diagnostic could be found which would allow one to determine which clusters were undergoing mergers, and which would quantify the strength of the merger (e.g., the rate of energy input from merger shocks). Then, merging clusters could either be culled from the cluster samples, or their properties could be corrected for the effects of mergers. This is difficult to do based on either X-ray or SZ observations alone. For example, it is difficult to detect mergers occurring along our line-of-sight. If possible mergers in clusters are detected by using the differences of their X-ray or SZ properties from standard scaling relations, and these clusters are removed from samples, this will tend to bias the results. What would be ideal would be to find a 'Merge-o-Meter' - some diagnostic for mergers that did not rely on their X-ray or SZ properties.

In principle, low frequency, diffuse radio emission (from radio halos and relics) might provide such a diagnostic for cluster mergers for cosmology and dark energy studies. Low frequency radio surveys of clusters could be used to either remove merging clusters from relaxed cluster samples for cosmology, or to attempt to correct the cluster X-ray and/or SZ properties for the boosts produced by mergers. One might expect that the populations of the relativistic electrons generated by mergers through shock or turbulent acceleration would increase in proportion to the energy input for the merger; this connection could provide a physical basis for correcting the X-ray and/or SZ properties for merger boosts.

\section{Acknowledgements}

I thank T. Clarke for useful comments. This work was supported by NASA through XMM-Newton awards NNG04GO34G, NNG04GO80G, NNG04GP46G, NNG05GA34G, NNG05GO50G, and NNG06GD54G, and through Chandra awards GO4-5133X, GO45137X, and GO5-6126X.

\section{References}

Blanton, E. L., Sarazin, C. L., McNamara, B. R., \& Wise, M. W. 2001, ApJ (Letters), 558, L15 Cassano, R., \& Brunetti, G. 2005, MNRAS, 357, 1313

Clarke, T. E., \& Ensslin, T. A. 2006, AJ, 131, 2900

Ensslin, T. A., \& Gopal-Krishna 2001, A\& A, 366, 26

Feretti, L., Brunetti, G., Giovannini, G., Kassim, N., Orru, E., \& Setti, G. 2004, JKAS, 37, 315

Fujita, Y., Sarazin, C. L., Kempner, J. C., Rudnick, L., Slee, O. B., Roy, A. L., Andernach, H., \& Ehle, M. 2002, ApJ, 575, 764

Fujita, Y., Takizawa, M., \& Sarazin, C. L. 2003, ApJ, 584, 190

Giovannini, G., \& Feretti, L. 2004, JKAS, 37, 323

Mohr, J. J. 2005, in: S. C. Wolff, \& T. R. Lauer (eds.), Observing Dark Energy, ASP-CS, 339, 140

Randall, S. W., Sarazin, C. L., \& Ricker, P. M. 2002, ApJ, 577, 579

Ricker, P. M., \& Sarazin, C. L. 2001 ApJ, 561, 621

Wik, D. R., Sarazin, C. L., Ricker, P. M., \& Randall, S. W. 2006, in preparation [see also: BAAS 38, 371, \#9, \#13.33 